\title{
Low Cycle Fatigue Behavior of a 10\% Cr Martensitic Steel at $600^{\circ} \mathrm{C}$
}

\author{
Roman MISHNEV, Nadezhda DUDOVA* and Rustam KAIBYSHEV \\ Belgorod State University, Pobeda 85, Belgorod, 308015 Russia. \\ (Received on June 6, 2015; accepted on August 3, 2015; J-Stage Advance published date: September \\ 8, 2015)
}

\begin{abstract}
In this work, the low cycle fatigue (LCF) behavior of a $10 \% \mathrm{Cr}$ martensitic steel was studied under fully reversed tension-compression loading at $600^{\circ} \mathrm{C}$ for constant strain amplitudes between \pm 0.20 to $\pm 1.0 \%$. The cyclic stress response exhibited a gradual softening regime until fracture after an initial short period of a nearly stable stress. The fatigue lifetime curve at $600^{\circ} \mathrm{C}$ was determined to be described by the Basquin-Manson-Coffin relationship. At constant strain amplitudes of $\geq \pm 0.35 \%$, the cyclic strain resistance of the steel mostly depends on its plastic properties. The cyclic softening effect was attributed to the coarsening of the laths and subgrains, accompanied by a decrease in the dislocation density.
\end{abstract}

KEY WORDS: martensitic steel; low cycle fatigue; microstructure; dislocation; precipitation.

\section{Introduction}

Heat-resistant martensitic steels are widely used as materials in various critical components of the fossil power plants due to their superior creep resistance and high temperature strength under monotonic loading. ${ }^{1)}$ A recently developed $10 \% \mathrm{Cr}$ steel with $\mathrm{Co}, \mathrm{W}$, Mo additives and increased $\mathrm{B}$ content is a new-generation of steels for use in components of steam turbines, such as rotors and blades, exhibiting improved creep properties compared to commercial P92 steel. ${ }^{2,3)}$ Superior creep strength of this steel is attributed to the high coarsening resistance of $\mathrm{M}_{23} \mathrm{C}_{6}$ located at the lath/(sub)grain boundaries and uniformly distributed MX precipitates during long-term aging and under creep conditions. $^{3,4)}$ This dispersion of secondary phase particles effectively stabilizes the tempered martensite lath structure (TMLS) under exploitation conditions. ${ }^{3)}$

Steam turbine components are often subjected to pulsating centrifugal forces and repeated temperature gradients, which generate strong fatigue conditions and can lead to low cycle fatigue (LCF) damage. ${ }^{5)}$ Approximately $65 \%$ of the damage in massive turbine cylinders and valve chambers are associated with $\mathrm{LCF}^{6)}$ due to cyclic thermal stresses from start-up and shut-down. Advanced creep-resistant steels must withstand LCF. Therefore, LCF behavior at service relevant load changing conditions is an important characteristic for the qualification of this steel for high temperature applications. ${ }^{5)}$ Unfavorable changes in the TMLS, namely, lath/subgrain coarsening and a decrease in the dislocation density may occur in $9 \%$ to $12 \% \mathrm{Cr}$ steels during cyclic loading, leading to cyclic softening and a drastic deterioration in the creep strength. ${ }^{7,8)}$ However, the cyclic behavior

\footnotetext{
* Corresponding author: E-mail: dudova@bsu.edu.ru

DOI: http://dx.doi.org/10.2355/isijinternational.ISIJINT-2015-336
}

of the new generation of martensitic steels containing low $\mathrm{N}$ has not been investigated in sufficient detail. In this work, an advanced $10 \% \mathrm{Cr}$ steel was tested at a temperature of $600^{\circ} \mathrm{C}$. The influence of constant strain amplitude on the low cycle fatigue properties and the microstructure changes during cyclic loading was studied to elucidate the resistance of this steel during cyclic loading and produce a better understanding of the damage caused by LCF.

\section{Material and Experimental Procedure}

A $10 \%$ Cr steel with the chemical composition (in wt.\%) $0.1 \% \mathrm{C}, 0.06 \% \mathrm{Si}, 0.1 \% \mathrm{Mn}, 10.0 \% \mathrm{Cr}, 0.17 \% \mathrm{Ni}, 0.7 \%$ Mo, $0.05 \% \mathrm{Nb}, 0.2 \% \mathrm{~V}, 0.003 \% \mathrm{~N}, 0.008 \% \mathrm{~B}, 2.0 \% \mathrm{~W}$, $3.0 \% \mathrm{Co}, 0.002 \% \mathrm{Ti}, 0.006 \% \mathrm{Cu}, 0.01 \% \mathrm{Al}$ and $\mathrm{Fe}-$ balance was examined. This $10 \% \mathrm{Cr}$ steel was produced as hotforged strip with cross-sectional dimensions of $65 \times 140$ $\mathrm{mm}^{2}$. The steel was subjected to normalizing at $1060^{\circ} \mathrm{C}$ for $30 \mathrm{~min}$ followed by air cooling and final tempering at $770^{\circ} \mathrm{C}$ for $3 \mathrm{~h}$.

Tensile tests were carried out using flat specimens with a gauge length of $35 \mathrm{~mm}$ and a cross-section of $7 \mathrm{~mm} \times 3$ $\mathrm{mm}$ using an Instron 5882 testing machine at temperatures of 20 and $600^{\circ} \mathrm{C}$ with a strain rate of $2 \times 10^{-3} \mathrm{~s}^{-1}$. Low cycle fatigue tests were carried out at $600^{\circ} \mathrm{C}$ under fully reversed tension-compression loading conditions with different constant total strain amplitudes $\left(\varepsilon_{\mathrm{ac}}=\right.$ const $)$ ranging from \pm 0.20 to $\pm 1.0 \%$ on cylindrical specimens with a gauge length of $18 \mathrm{~mm}$ and a diameter of $5 \mathrm{~mm}$ using an Instron 8801 testing machine. The ratio of minimum strain to maximum strain (R) was -1 and the frequency was $0.5 \mathrm{~Hz}$. Stressstrain hysteresis loops were recorded continuously to determine the cycle dependent changes in the stress and plastic strain amplitude. The characteristic parameters of the loop were determined for half-life cycles to failure $\left(\mathrm{N}=0.5 \mathrm{~N}_{\mathrm{f}}\right)$ to 
evaluate the basic fatigue properties of the steel.

The microstructure was examined using a Quanta 600FEG scanning electron microscope equipped with an electron back scatter diffraction (EBSD) pattern analyzer incorporating an orientation imaging microscopy (OIM) system and a Jeol JEM-2100 transmission electron microscope (TEM) with an INCA energy dispersive X-ray spectrometer. TEM foils were prepared by double jet electro-polishing using a solution of $10 \%$ perchloric acid in glacial acetic acid. The OIM images were obtained with a step size of 120 to 170 $\mathrm{nm}$ and subjected to a cleanup procedure, setting a minimal confidence index of 0.1 and setting a minimal point number per grain of 8 . The distance between the high-angle boundaries with misorientations greater than $15^{\circ}$ was measured on the OIM images. Low- and high-angle boundary densities were calculated as the total length of the boundaries per unit area on the OIM images. The transverse lath/subgrain sizes were measured on the TEM micrographs by the linear intercept method counting all the clear visible (sub)boundaries. The dislocation densities were estimated by counting the individual dislocations in the (sub)grain/lath interiors.

\section{Experimental Results}

\subsection{Initial Microstructure}

The TMLS of the $10 \% \mathrm{Cr}$ steel subjected to the heat treatment is characterized by an average size of prior austenite grains (PAG) of $\sim 35 \mu \mathrm{m}$, an average distance between highangle boundaries (HAB) of $\sim 3.5 \mu \mathrm{m}$, and an average width of martensite laths of $\sim 0.4 \mu \mathrm{m}$ (Fig. 1). The angular misorientations of the boundaries within a packet did not exceed $10^{\circ}$, and the cumulative (point-to-origin) misorientation in the interiors of this typical packet is nearly independent of the distance because of mutually compensated block rotations (Fig. 1(a)). The dislocation density within the lath interiors was $\sim 1.7 \times 10^{14} \mathrm{~m}^{-2}$. Thus, there exists a set of well-defined 2D dislocation arrays in the steels, which may be interpreted in terms of incidental dislocation boundaries because no cumulative misorientation ${ }^{9)}$ is observed across the laths. These dislocation networks contain a large density of separate dislocations within their interiors. In addition, numerous nanoscale $\mathrm{M}_{23} \mathrm{C}_{6}$-type carbides with a mean size of $70 \mathrm{~nm}$ are located at the boundaries of PAGs/packets/ blocks/laths (Fig. 1(b)). Nb- and V-enriched MX-type carbonitrides with sizes of $30 \mathrm{~nm}$ and $40 \mathrm{~nm}$, respectively, were uniformly distributed within the martensitic laths. ${ }^{3)}$ Therefore, the steel examined in this work as other high $\mathrm{Cr}$ martensitic steels should exhibit a unique cyclic stress-strain response compared to well-annealed materials.

\subsection{Tensile Properties}

The engineering stress-strain curve is presented in Fig. 2(a) and the yield stress (YS), ultimate tensile strength (UTS), ductility, and ratio of UTS/YS obtained in tension at room temperature and $600^{\circ} \mathrm{C}$ are shown in Table 1. After the short stage of initial strain hardening, the peak stress is attained. Further deformation is accompanied by significant strain softening. A UTS/YS ratio of 1.07 at a temperature of $600^{\circ} \mathrm{C}$ predicts the cyclic softening behavior of the $10 \% \mathrm{Cr}$ steel because this material exhibits no the strain-hardening ability in tension, which is in contrast to the cyclic stabiliz-
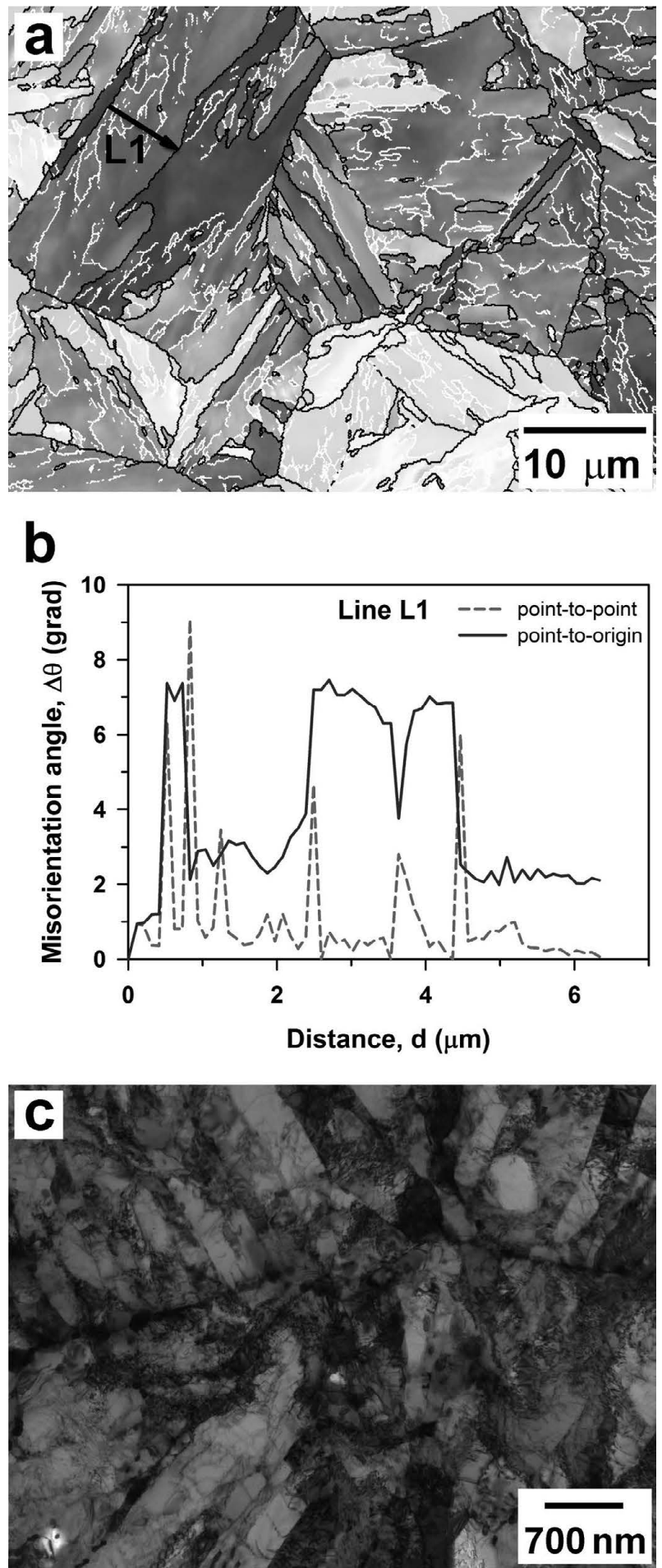

Fig. 1. Typical tempered martensite lath structure of the $10 \% \mathrm{Cr}$ steel: (a) OIM picture, the white and black lines correspond to boundaries with misorientations of $2^{\circ} \leq \theta<15^{\circ}$ and $15^{\circ} \leq \theta$, respectively, (b) Point-to-point and point-toorigin misorientations along the line L1, and (c) Typical TEM micrograph.

ing behavior at room temperature predicted by the UTS/YS ratio of 1.25 .

\subsection{LCF Behavior}

The cyclic stress response of the $10 \% \mathrm{Cr}$ steel at $600^{\circ} \mathrm{C}$ shown in Fig. 2(a) is strongly dependent on the strain amplitude. The number of cycles to failure decreases from $1.7 \times 10^{4}$ to $2 \times 10^{2}$ as the strain amplitude increases from 

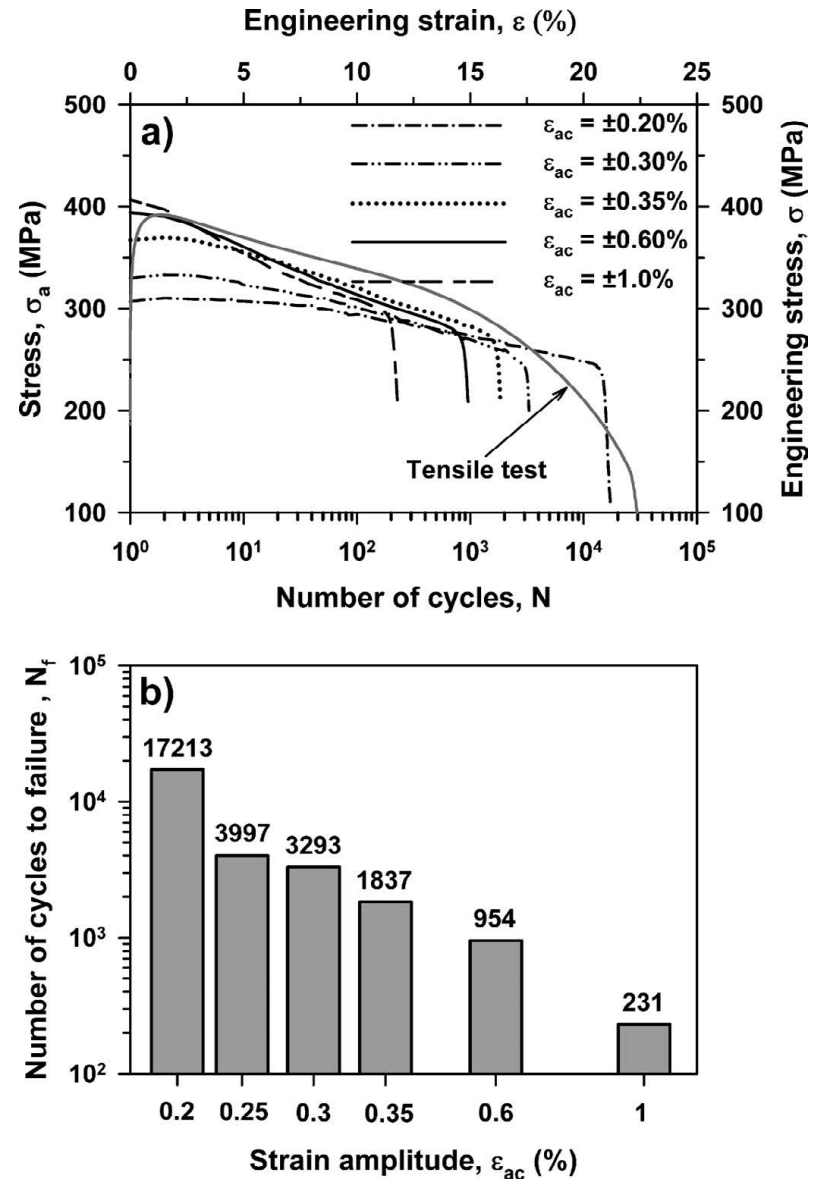

Fig. 2. Cyclic stress response curves (a) and fatigue life (b) of the $10 \% \mathrm{Cr}$ steel at $600^{\circ} \mathrm{C}$ for different strain amplitudes from $\pm 0.20 \%$ to $\pm 1.0 \%$.

Table 1. Mechanical properties of the tempered $10 \% \mathrm{Cr}$ steel at room temperature and $600^{\circ} \mathrm{C}$.

\begin{tabular}{ccccc}
\hline $\mathrm{T}\left[{ }^{\circ} \mathrm{C}\right]$ & YS $[\mathrm{MPa}]$ & UTS $[\mathrm{MPa}]$ & El. $[\%]$ & UTS/YS \\
\hline 20 & 560 & 700 & 16.2 & 1.25 \\
600 & 365 & 390 & 23.1 & 1.07 \\
\hline
\end{tabular}

\pm 0.20 to $\pm 1 \%$ (Fig. 2(b)), which is typical for martensitic heat-resistant steels. ${ }^{10)}$ As with other martensitic steels, ${ }^{7}$ the $10 \% \mathrm{Cr}$ steel exhibits continuous softening to failure.

Three strain amplitude intervals can be distinguished by cyclic softening behavior. First, at small strain amplitudes $\varepsilon_{\mathrm{ac}}= \pm 0.2 \%$, during the initial period of the first 1 to $10^{2}$ cycles, a nearly stable stress response stage is observed. Remarkable softening with a coefficient, $\Delta \sigma / \Delta \operatorname{lgN} \mathrm{f}_{\mathrm{f}}$, of approximately 18 appears after $10^{2}$ cycles and occurs up to failure (Fig. 2(a)). This type of cyclic behavior results in relatively high fatigue life larger than $10^{4}$ cycles (Fig. 2(b)). Second, at strain amplitudes of $\pm(0.3-0.35) \%$, a short period ( $1-10$ cycles) of stress amplitude stabilization is followed by a cyclic softening with the coefficient, $\Delta \sigma / \Delta \operatorname{lgN} \mathrm{f}_{\mathrm{f}}$, of $\sim 37$. This cyclic behavior provides the number of cycles to failure less than $10^{4}$ (Fig. 2(b)). Therefore, increase in total strain amplitude from low $( \pm 0.2 \%)$ to medium $( \pm 0.35 \%)$ provides $+106 \%$ increase in the cyclic softening coefficient and about ten-fold decrease in the fatigue life. Third, at large strain amplitudes of \pm 0.6 and $\pm 1.0 \%$, the steel softens immediately after first cycle and the cyclic softening coefficient of $\sim 45$ is high. The monotonic and cyclic stress-strain curves at high strain amplitudes are nearly the same (Fig. 2(a)) and, therefore, softening under such high amplitude and tension occurs in nearly the same manner due to similar changes in the TMLS. This cyclic softening behavior corresponds with low fatigue life less than 1000 cycles. Thus, increase in total strain amplitude from medium $( \pm 0.3 \%)$ to high $( \pm 0.6 \%)$ leads to $+21 \%$ increase in the cyclic softening coefficient and about two-fold decrease in the fatigue life.

Figure 3 shows the hysteresis loops recorded during the tests for the low $( \pm 0.2 \%)$, medium $( \pm 0.35 \%)$, and high $( \pm 0.6 \%)$ total strain amplitude levels. During the LCF tests at $600^{\circ} \mathrm{C}$, the values of the stress amplitude decrease and the values of the plastic component of strain amplitude increase with the number of cycles, indicating continuous cyclic softening. Meanwhile, the stress amplitude at the half-life cycle does not change significantly with increases in the strain amplitude (Fig. 3(d)). The three-fold increase in the strain amplitude from \pm 0.2 to $\pm 0.6 \%$ leads to only a $+15 \%$ increase in the stress amplitude, despite being accompanied by an approximate decrease in the fatigue life by a factor of 18 from 17213 to 954 cycles (Figs. 2, 3).

The fatigue life of the steel may be described by the Basquin-Manson-Coffin relationship: ${ }^{11)}$

$$
\varepsilon_{a c}=\varepsilon_{a e}+\varepsilon_{a p}=\frac{\sigma_{f}^{\prime}}{E}\left(2 N_{f}\right)^{b}+\varepsilon_{f}^{\prime}\left(2 N_{f}\right)^{c},
$$

where $\varepsilon_{\mathrm{ac}}, \varepsilon_{\mathrm{ae}}$, and $\varepsilon_{\mathrm{ap}}$ are the total strain amplitude, elastic strain amplitude and plastic strain amplitude, respectively, $2 N_{f}$ is the number of reversals to failure, $\sigma_{f}$ is the fatigue strength coefficient, $\varepsilon_{f}^{\prime}$ is the fatigue ductility coefficient, $b$ is the fatigue strength exponent, and $c$ is the fatigue ductility exponent.

The evolution of the plastic strain amplitude, $\varepsilon_{\mathrm{ap}}$, elastic strain amplitude, $\varepsilon_{\mathrm{ae}}$, and the total strain range, $\varepsilon_{\mathrm{ac}}$, as functions of the number of cycles to failure are plotted in Fig. 4(a) using a double logarithmic scale and the parameters of Eq. (1) are presented in Table 2. The dependences of $\varepsilon_{\mathrm{ae}}$ and $\varepsilon_{\mathrm{ap}}$ on the number of reversals to failure, $2 \mathrm{~N}_{\mathrm{f}}$, are linear. The value of the fatigue strength exponent, $b$, obtained by the method of least squares, is -0.058 and the value of $c$, the fatigue ductility exponent, is -0.66 . The point of intersection between the two curves $\left(\varepsilon_{\mathrm{ae}}\right.$ vs $\left.2 \mathrm{~N}_{\mathrm{f}}\right)$ and $\left(\varepsilon_{\mathrm{ap}}\right.$ vs $2 \mathrm{~N}_{\mathrm{f}}$ ) corresponds to 5339 reversals. At this intersection point, the level of total strain amplitude is about $\pm 0.35 \%$. Therefore, at high total strain amplitudes $> \pm 0.35 \%$, the plastic strain plays a major role in cyclic strain softening, whereas, for low total strain amplitude less than $\pm 0.35 \%$, the cyclic strain resistance is affected by elastic deformation processes. The transition in the shape of the cyclic stress response curves (Fig. 2(a)) and the stress-strain hysteresis loops (Fig. 3(d)) occurring at a medium total strain amplitude of $\pm 0.35 \%$ supports this conclusion.

The half-life cycle stress amplitude plastic strain amplitude curve may be represented by the following power law relationship: ${ }^{12}$

$$
\sigma_{a}=K^{\prime}\left(\varepsilon_{a p}\right)^{n^{\prime}} \Rightarrow \lg \sigma_{a}=\lg K^{\prime}+n^{\prime} \lg \varepsilon_{a p},
$$

where $\mathrm{K}^{\prime}$ is the cyclic strength coefficient and $\mathrm{n}^{\prime}$ is the 

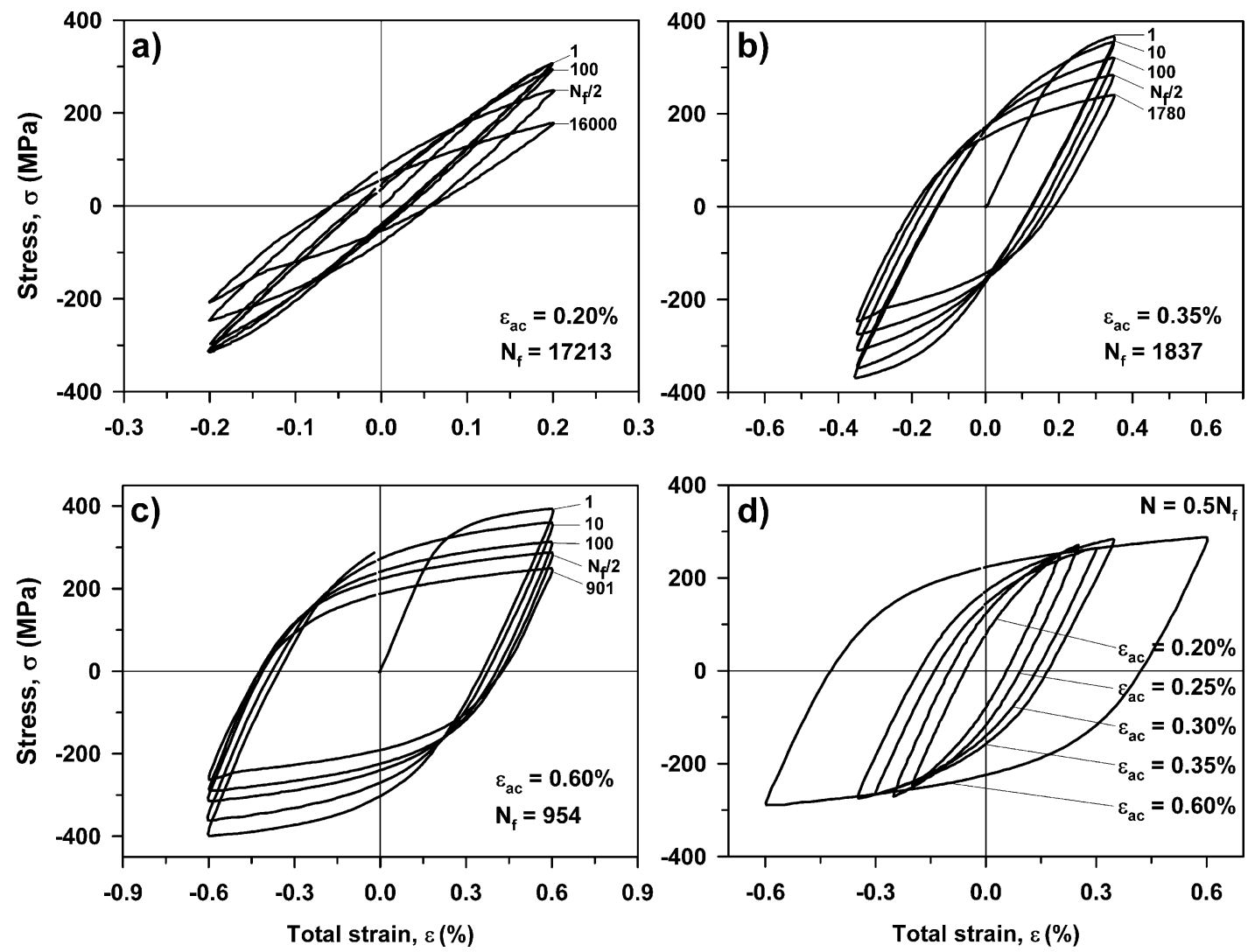

Fig. 3. Change in the stress-strain hysteresis loop as the number of cycles increase for the $10 \% \mathrm{Cr}$ steel at $600{ }^{\circ} \mathrm{C}$ at a total strain amplitude $\varepsilon_{\mathrm{ac}}$ of: (a) $\pm 0.2 \%$, (b) $\pm 0.35 \%$, and (c) $\pm 0.6 \%$. (d) Hysteresis loops for the half-life cycle at a total strain amplitude $\varepsilon_{\mathrm{ac}}$ from $\pm 0.20 \%$ to $\pm 0.6 \%$.
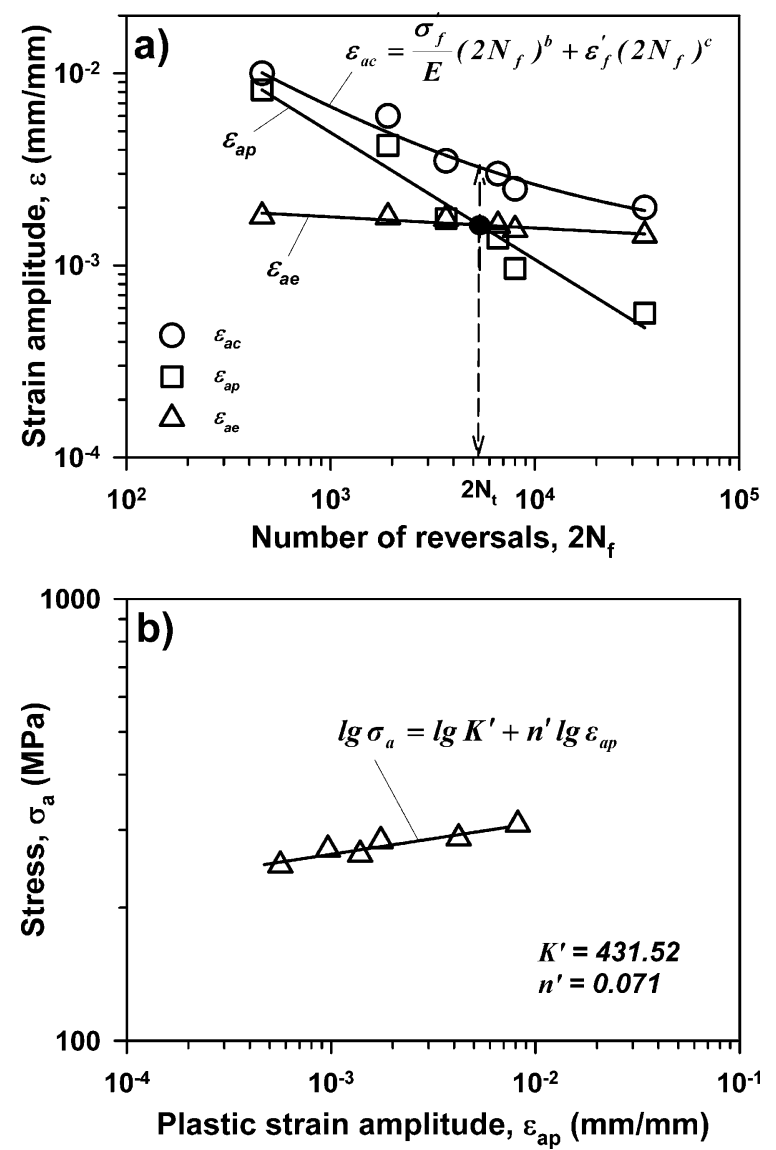

Fig. 4. Fatigue life as a function of plastic and elastic strain amplitudes (a) and cyclic stress resistance (b) of the $10 \%$ $\mathrm{Cr}$ steel at $600^{\circ} \mathrm{C}$.
Table 2. Low cycle fatigue parameters of the $10 \% \mathrm{Cr}$ steel at $600^{\circ} \mathrm{C}$.

\begin{tabular}{ccccc}
\hline$\sigma_{\mathrm{f}}^{\prime} / \mathrm{E}$ & $\mathrm{b}$ & $\varepsilon_{\mathrm{f}}^{\prime}$ & $\mathrm{c}$ & $2 \mathrm{~N}_{\mathrm{t}}$ \\
\hline 0.00267 & -0.05801 & 0.4736 & -0.6612 & 5339 \\
\hline
\end{tabular}

cyclic strain hardening exponent. The corresponding relationship is shown in Fig. 4(b). The values of $\mathrm{K}^{\prime}$ and $\mathrm{n}^{\prime}$, determined using the method of least squares, are 431.5 and 0.071 , respectively. These data are in a good agreement with fatigue behavior of other high-chromium martensitic steels. $^{6,13,14)}$

\subsection{Fracture Behavior}

Observations of the fracture surfaces show that the appearance of the fatigue fractures depends on the values of the strain amplitude (Fig. 5). At $\varepsilon_{\mathrm{ac}}= \pm 0.2 \%$, a single crack is initiated at an inclusion near the surface (Fig. 5(a)), whereas several crack initiation sites are observed in the specimen tested at $\varepsilon_{\mathrm{ac}}= \pm 0.6 \%$ (Fig. 5(b)). In general, Stage I is poorly defined, as with most materials subjected to low cycle high-stress fatigue. ${ }^{15)}$ The largest portion of fatigue fracture is Stage II crack growth, which occurs via transgranular fracture and comprises approximately $64 \%$ and $47 \%$ of the strain amplitudes for $\varepsilon_{\mathrm{ac}}= \pm 0.2 \%$ and $\varepsilon_{\mathrm{ac}}= \pm 0.6 \%$, respectively. The $10 \% \mathrm{Cr}$ steel exhibits a high oxidation resistance that results in smooth and poorly defined features on the fatigue fracture surface (Figs. 5(c), 5(d)) and is attributed to nearly complete slip reversal. ${ }^{15)}$ Propagation of the fatigue 

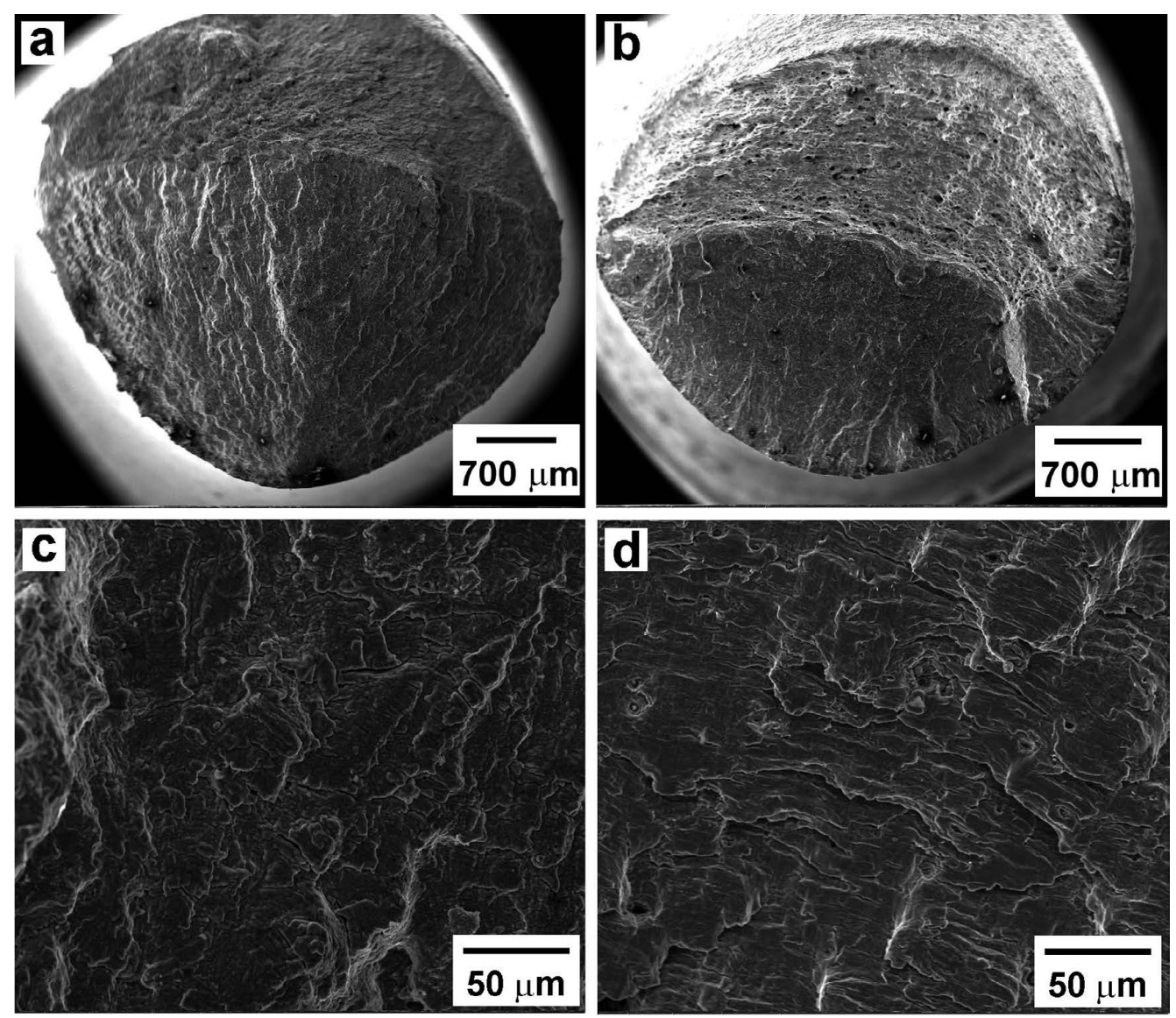

Fig. 5. SEM micrographs of the fracture surfaces for the $10 \% \mathrm{Cr}$ steel tested at $600^{\circ} \mathrm{C}$ and a total strain amplitude of $\pm 0.2 \%$ ( $\mathrm{a}$ and $\mathrm{c}$ ) and $\pm 0.6 \%$ (b and $\mathrm{d}$ ).
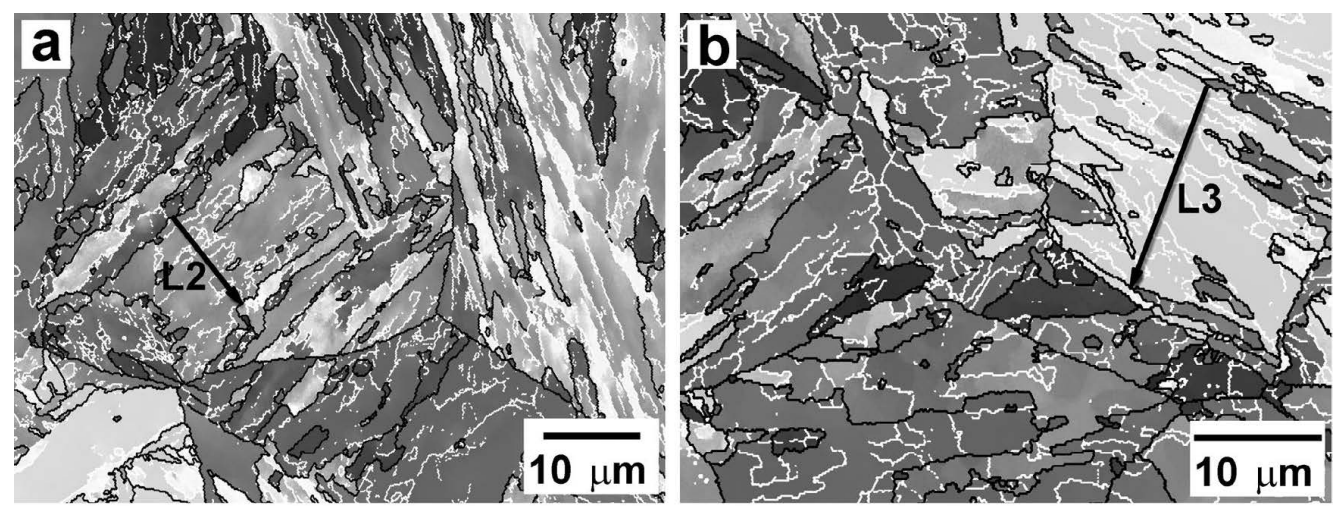

C

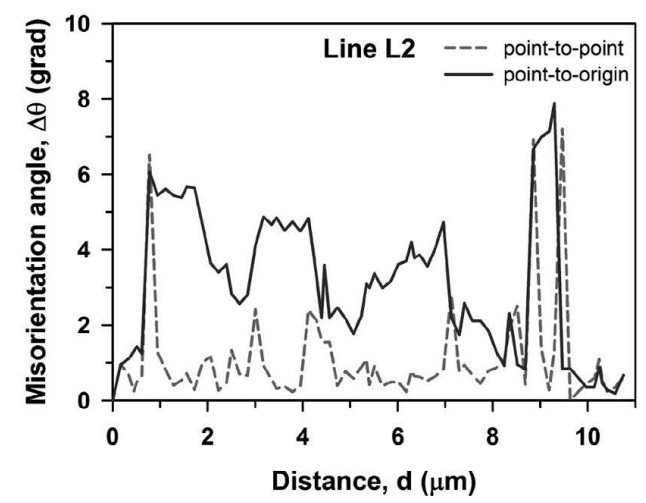

d

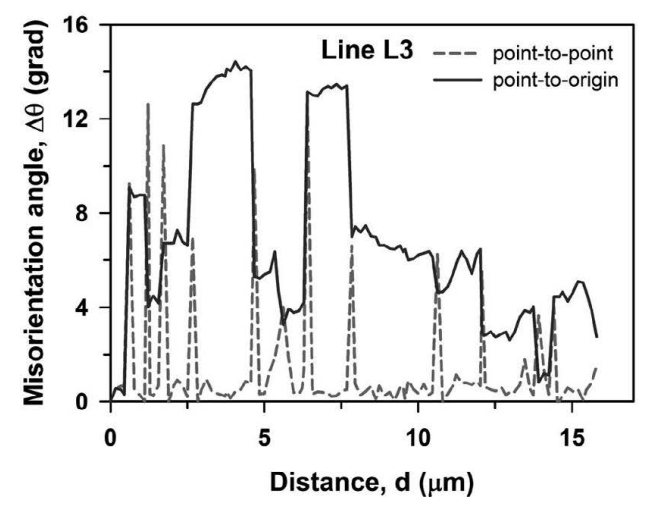

Fig. 6. OIM pictures (a and b) and misorientations along lines L2 (c) and L3 (d) of the 10\% Cr steel after fatigue failure for different total strain amplitudes of: $\pm 0.2 \%$ (a and c) $-\mathrm{N}_{\mathrm{f}}=17213$ cycles; $\pm 0.6 \%$ (b and d) $-\mathrm{N}_{\mathrm{f}}=954$ cycles. 
crack during Stage II is strongly affected by the portion of plastic strain in the total amplitude and the magnitude of the alternating stress. At $\varepsilon_{\mathrm{ac}}= \pm 0.2 \%$, crack propagation requires the stress concentration to confine the dislocation slip to a small region at the tip of the crack due to the relatively low stress magnitude as the remainder of the material is subjected to elastic strain. ${ }^{15)}$ As a result, the propagation of the fatigue crack front occurs by local shift of its parts. Short and closely spaced striations joined by tear ridges are seen to evolve (Fig. 5(c)). The fracture surface exhibits a sawtooth profile. It is apparent that the crack propagation can be easily arrested and crack propagation resumes only after a certain number of cycles.

In contrast, at $\varepsilon_{\mathrm{ac}}= \pm 0.6 \%$, extended and widely spaced striations are observed, which exhibit slip traces on their edges (Fig. 5(d)). Increasing the magnitude of the alternating stress facilitates the propagation of the fatigue crack. The profile of the fatigue fracture becomes flat. The wave shape of the striations, their concavity, and the formation of dimples around the inclusions are indicative of a high rate of crack propagation. Most secondary phase particles remain intact after crack propagation, decreasing the local crack growth rate; in addition, crack branching is observed (Figs. 5(c) and 5(d)). Thus, transition from the low $( \pm 0.2 \%)$ to high $( \pm 0.6 \%)$ total strain amplitude levels highly facilitates the propagation of the fatigue crack during Stage II. It is apparent that effect of the strain amplitude on the fatigue life is attributed to the promotion of crack propagation by plastic deformation processes.

During Stage III the striation-forming mode is displaced by the static dimple rupture at any strain amplitudes (Figs. $5(\mathrm{a})$ and $5(\mathrm{~b}))$. At $\varepsilon_{\mathrm{ac}}= \pm 0.2 \%$, quasi-cleavage fracture is observed in the transition zone. Small dimples dominate in the static fracture zone. At $\varepsilon_{\mathrm{ac}}= \pm 0.6 \%$, the transition zone is revealed by the appearance of large and deep dimples with a rounded shape between the striations on the flat fracture surface. Some of these contain coarse inclusions on their bottom. A high portion of small dimples appear in the static fracture zone.

\subsection{Microstructural Observation}

Figures 6 and 7 show the microstructure of the steel after fatigue testing with different total strain amplitudes $\left(\varepsilon_{\mathrm{ac}}\right)$ of $\pm 0.2 \%$ and $\pm 0.6 \%$. The microstructural parameters
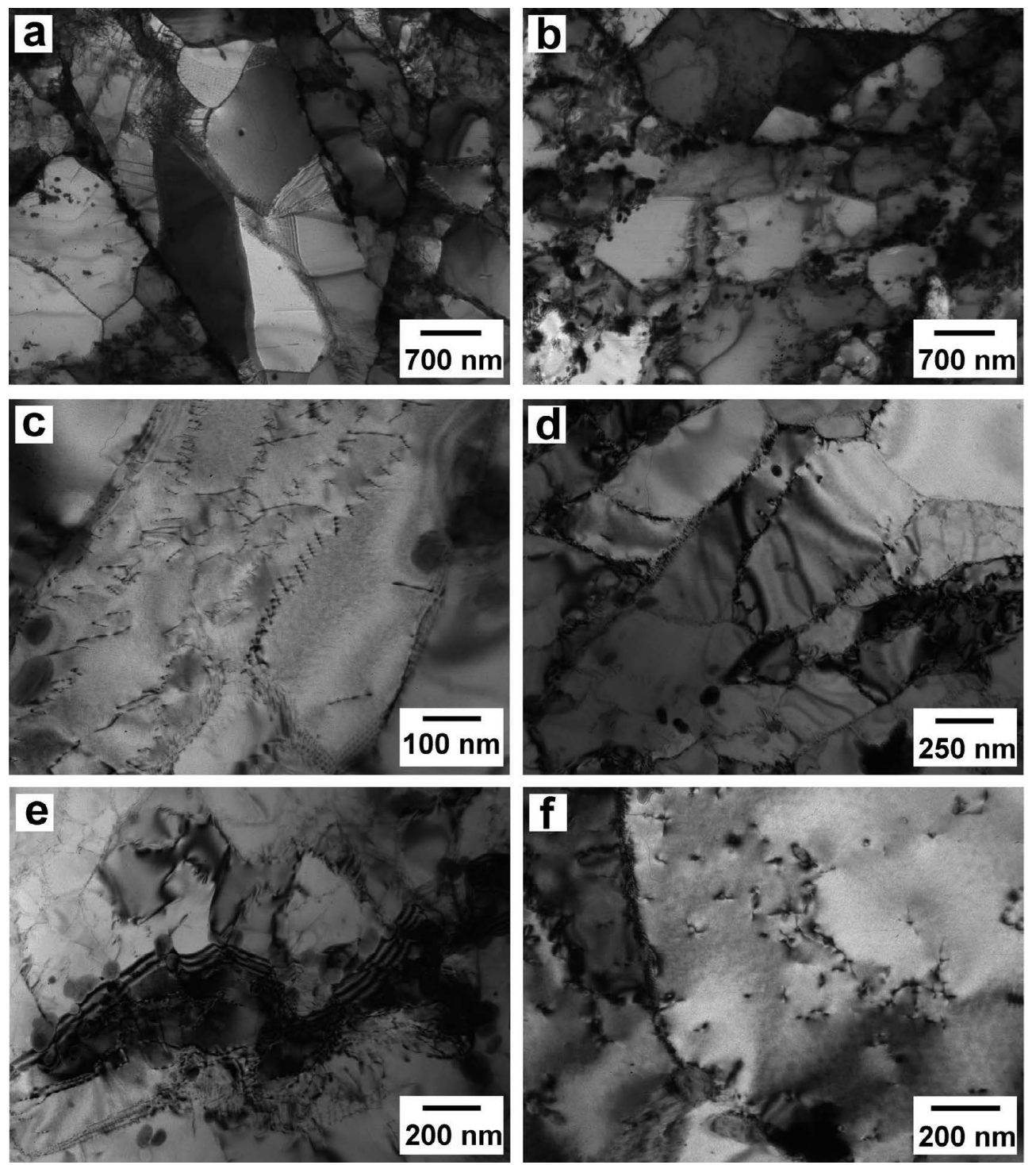

Fig. 7. TEM micrographs of the $10 \% \mathrm{Cr}$ steel after fatigue failure for different total strain amplitudes of: $\pm 0.2 \%$ (a, $\mathrm{c}$, and e) $-\mathrm{N}_{\mathrm{f}}=17213$ cycles; $\pm 0.6 \%(\mathrm{~b}, \mathrm{~d}$, and $\mathrm{f})-\mathrm{N}_{\mathrm{f}}=954$ cycles. 
are listed in Table 3. The TMLS could not withstand the cyclic deformation. The lath structure loses stability during cyclic loading and tends to transform continuously to a subgrain structure. Higher strain amplitude promotes this transition and, therefore, increasing the portion of the plastic strain in the total $\varepsilon_{\mathrm{ac}}$ value promotes the formation of subgrain structure and a decrease of dislocation density. The subgrain size increased from $0.4 \mu \mathrm{m}$ to $0.5 \mu \mathrm{m}$ and 0.9 $\mu \mathrm{m}$ under low and high cyclic strain amplitudes of $\varepsilon_{\mathrm{ac}}= \pm 0.2$ and $\pm 0.6 \%$, respectively (Figs. $7(\mathrm{a})$ and $7(\mathrm{~b})$ ). The cyclic softening effect in the $10 \% \mathrm{Cr}$ steel is correlated to the transformation of the initial martensitic laths to subgrains and to a decrease of the dislocation density as in other high $\mathrm{Cr}$ steels. ${ }^{7,16)}$ The microstructural evolution for material exposed to LCF occurs in two main ways. First, 2D dislocation arrays convert to 3D networks of subgrain boundaries (Figs. 7(a) and 7(b)) that are accompanied by a decrease in the fraction of low-angle $\left(2-15^{\circ}\right)$ boundaries (LAB) as well as the density of LABs per unit area (Table 3 ). However, the portion of LABs with a high misorientation of $\geq 6^{\circ}$ increases (Fig. 6). It appears that the disappearance of some low angle boundaries with a low misorientation occur due to coalescence and/or joining to HABs. Most of subgrains acquired an almost equiaxed shape (Figs. 7(a) and 7(b). Second, dynamic recovery ${ }^{17)}$ takes place. The dislocation rearrangements resulted in a decrease in the dislocation density within the lath interiors to approximately $1.2 \times 10^{14}$ and $0.6 \times 10^{14} \mathrm{~m}^{-2}$ after fatigue testing with $\varepsilon_{\mathrm{ac}}= \pm 0.2 \%$ and $\varepsilon_{\mathrm{ac}}= \pm 0.6 \%$, respectively (Table 3 ). The dislocations tended to rearrange in pile-ups along straight boundaries and form dislocation tangles near the grain boundary carbides during cyclic stress conditions (Figs. 7(c) and 7(d)). The misorientation across the laths in the block interiors retain their mutually compensated character as suggested by the point-to-origin misorientation variations along lines L2 and L3 in Fig. 6. Therefore, both processes occur through such internal reactions as the emission of lattice dislocations by Frank-read sources, rearrangement of lattice dislocations, and their mutual annihilation within the initial TMLS. No input from external dislocations to the existing dislocation arrays takes place. The density of the HAB length per unit area did not change during cyclic deformation and comprised approximately $7.0 \times 10^{5} \mathrm{~m}^{-1}$. Therefore, there is no effect from cyclic loading on the HABs.

The chains of $\mathrm{M}_{23} \mathrm{C}_{6}$ carbides restrained the subgrain boundary migration at a low strain amplitude of $\pm 0.2 \%$. The subgrain/lath boundary migration between the precipitates led to their bulging and irregular shape (Fig. 7(e)). Detachment of the boundaries from the carbides occurred at a high strain amplitude of $\pm 0.6 \%$, which resulted in the presence

Table 3. Effect of strain amplitude on the structural parameters of the $10 \%$ Cr steel.

\begin{tabular}{ccccc}
\hline & $\begin{array}{c}\text { Subgrain } \\
\text { size }[\mu \mathrm{m}]\end{array}$ & $\begin{array}{c}\text { Dislocation } \\
\text { density } \\
\times 10^{14}\left[\mathrm{~m}^{-2}\right]\end{array}$ & $\begin{array}{c}\text { Fraction of } \\
\text { LAB [\%] }\end{array}$ & $\begin{array}{c}\text { Density of } \\
\text { LAB, } \times 10^{5} \\
{\left[\mathrm{~m}^{-1}\right]}\end{array}$ \\
\hline Before tests & 0.4 & 1.7 & 58.1 & 10.0 \\
$\varepsilon_{\mathrm{ac}}= \pm 0.2 \%$ & 0.5 & 1.2 & 54.9 & 8.28 \\
$\varepsilon_{\mathrm{ac}}= \pm 0.6 \%$ & 0.9 & 0.6 & 50.2 & 7.34 \\
\hline
\end{tabular}

of chains of carbides in the subgrain interiors behind the lath boundaries (Fig. 7(b)). Therefore, an increase of a portion of the plastic strain in the strain amplitude highly increases driving pressure acting on low-angle boundaries, since Zener drag force is independent on the strain amplitude and exerted by a dispersion of the carbides and carbonitrides. ${ }^{18-20)}$

Pinning of the dislocations by small MX carbonitrides within the lath and subgrain interiors was also observed (Fig. 7(f)). The mean size of the precipitates (approximately $75 \mathrm{~nm}$ ) did not change during the LCF tests. Therefore, the boundary $\mathrm{M}_{23} \mathrm{C}_{6}$ carbides and $\mathrm{MX}$ carbonitrides located within the ferritic matrix hinders the transformation of the $2 \mathrm{D}$ lath structure to the 3D subgrains and rearrangements of lattice dislocations in the similar way when exposed to creep condition taking into account that under LCF the distribution of the carbides and carbonitrides remains unchanged. ${ }^{18,19)}$

\section{Discussion}

The 10\% Cr steel exhibits cyclic softening when exposed to cyclic loading as well as when exposed to tension at $600^{\circ} \mathrm{C}$. The TMLS of this steel, which is strengthened by $\mathrm{M}_{23} \mathrm{C}_{6}$ carbides and $\mathrm{MX}$ carbonitrides, was revealed to be unstable during low cycle fatigue testing, similar to other $9 \%$ to $12 \%$ Cr martensitic steels. ${ }^{7,16)}$ The TMLS is not able to withstand even small plastic strain amplitudes and changes when the dominant contributor to the total strain amplitude is the elastic deformation. The magnitude of the total strain amplitude and the relation of the plastic and elastic amplitudes affect the evolution of the dislocation structure and fatigue behavior. At a total strain amplitude of greater than or equal to $\sim \pm 0.35 \%$, the plastic strain plays the dominant role and, therefore, the cyclic strain resistance of the steel depends on its plastic properties. Under such conditions, the transformation of the lath structure into a subgrain structure and the recovery of the dislocation structure occurs rapidly. In addition, high plastic strain amplitude highly facilitates the propagation of fatigue crack. Superposition of these two factors results in a low fatigue life under LCF condition with a high strain amplitude. For instance, at a total strain amplitude of $\pm 0.6 \%$, the plastic component $\left(\varepsilon_{a p}=0.4 \%\right)$ is larger than elastic component $\left(\varepsilon_{a e}=0.2 \%\right)$. Correspondingly, the fatigue life is less than 1000 cycles. The microstructural evolution providing the cyclic softening is similar to the microstructural changes observed for creep conditions at $\mathrm{T} \geq 550^{\circ} \mathrm{C}$. ${ }^{1,18)}$

However, a rather long fatigue life was observed during fatigue testing with a total strain amplitude below $\pm 0.35 \%$, or when the elastic strain component exceeded the plastic strain. A fatigue life of approximately 17000 cycles was recorded during fatigue tests with $\varepsilon_{\mathrm{ac}}= \pm 0.2 \%$, when the elastic and plastic strain components were $0.15 \%$ and $0.05 \%$, respectively. The structural changes at such cyclic conditions are associated with the Bauschinger effect, ${ }^{13,21,22}$ ) when decreasing the flow stress at the following cycles is attributed to the spilling of dislocation boundaries into separate dislocations ${ }^{13)}$ or to the reverse motion of the dislocations with respect to the lath boundary, followed by their annihilation. ${ }^{16,21)}$ The mutually compensated misorien- 
tations across the martensite blocks that were revealed by the structural observations (Figs. 1 and 6) suggest that the lath/subgrain boundaries are composed of dislocations with opposite Burgers vectors. During cyclic deformation in the forward and reverse directions, the moving dislocations may be captured by dislocation lath/subgrain boundaries, which serve as traps. Next, dislocation annihilation can take place if the distance between the dislocations at their interaction in the dislocation boundary is less than a critical value, which varies from $50 \mathrm{~nm}$ at room temperature for screw dislocations to $4 \mathrm{~nm}$ at $650^{\circ} \mathrm{C}$ for edge dislocations. ${ }^{16)}$ The annihilation processes result in decreasing the amount of lath boundary misorientation. When the boundary misorientation decreases to a threshold value of the pinning pressure exerted by second phase particles ${ }^{19,21)}$ the dislocation boundary starts to migrate or break into separate dislocations. This coalescence and trapping of mobile dislocations at separate, highly misoriented LABs leads to increase in the misorientation during cyclic loading.

The precipitates of the $\mathrm{M}_{23} \mathrm{C}_{6}$ and $\mathrm{MX}$ phases, which effectively stabilized the TMLS for long-term aging or creep conditions in low $\mathrm{N}$ steel ${ }^{3,4,23)}$ due to suppressing the lath boundary migration and dislocation rearrangements, are ineffective under cyclic stress conditions. The cyclic softening effect is correlated to the dislocation density decrease and subgrain coarsening.

It is noted that compared to P91- and P92-type steels and their modified versions such as GP91 and Eurofer 97 with conventional nitrogen contents of approximately $0.05 \%,{ }^{6,14)}$ the $10 \% \mathrm{Cr}$ steel with low $\mathrm{N}$ demonstrates nearly the same cyclic stress resistance at small-to-large strain amplitudes at a temperature of $600^{\circ} \mathrm{C}$. Slight improvements of fatigue life can be observed at small strain amplitudes less than $\pm 0.3 \%{ }^{6}{ }^{6}$ Therefore, modifications in the carbide and carbonitride distribution due to decreasing the nitrogen content and increasing the boron content ${ }^{3,4,23)}$ does not influence the resistance of the high $\mathrm{Cr}$ steels to cyclic softening.

\section{Summary}

In this work, we examined the low cycle fatigue (LCF) behavior and microstructure changes of a $10 \% \mathrm{Cr}$ steel using strain controlled cyclic testing at $600^{\circ} \mathrm{C}$. The primary results are summarized as follows:

1. The steel with a tempered martensite lath structure and strengthened by $\mathrm{M}_{23} \mathrm{C}_{6}$ carbides and $\mathrm{MX}$ carbonitrides shows continuous cyclic softening up to failure, although partial stabilization of the stress amplitude occurs at 1 to 100 cycles at a low strain amplitude of $\pm 0.2 \%$.

2. The LCF behavior at $600^{\circ} \mathrm{C}$ follows the BasquinManson-Coffin relationship. The plastic strain amplitude exceeds the elastic strain amplitude at total strain amplitudes of $\varepsilon_{a c}> \pm 0.35 \%$.

3. The transformation of the lath structure into a subgrain structure and a continuous decrease in the dislocation density take place under cyclic loading. These processes occur rapidly and result in a fatigue life of less than 2000 cycles at total strain amplitudes of $\pm 0.35 \%$ or larger.

\section{Acknowledgments}

The study was financially supported by the Russian Science Foundation, under grant No. 14-29-00173. The authors are grateful to the staff of the Joint Research Center, Belgorod State University, for their assistance with the instrumental analysis.

\section{REFERENCES}

1) K. Kimura, K. Sawada, H. Kushima and K. Kubo: Int. J. Mat. Res., 99 (2008), 395.

2) R. Viswanathan and W. Bakker: J. Mater. Eng. Perform., 10 (2001), No. 10, 96.

3) N. Dudova, R. Mishnev and R. Kaibyshev: Proc. 12th Int. Conf. on Creep and Fracture of Engineering Materials and Structures, Japan Institute of Metals, Sendai, (2012), B05.

4) N. Dudova, R. Mishnev and R. Kaibyshev: ISIJ Int., 51 (2011), 1912.

5) F. Abe, T.-U. Kern and R. Viswanathan: Creep Resistant Steels, Woodhead Publishing Limited and CRC Press LLC, Boca Raton, Fl, (2008), 678.

6) G. Golanski and S. Mrozinski: Eng. Fail. Anal., 35 (2013), 692.

7) B. Fournier, F. Dalle, M. Sauzay, J. Longour, M. Salvi, C. Caes, I. Tournie, P.-F. Giroux and S.-H. Kim: Mater. Sci. Eng. A, 528 (2011), 6934.

8) A. Sarkar, V. D. Vijayanand, P. Parameswaran, V. Shankar, R. Sandhya, K. Laha, M. D. Mathew, T. Jayakumar and K. E. Rajendra: Metall. Mater. Trans. A, 45 (2014), 3023.

9) D. A. Hughes, N. Hansen and D. J. Bammann: Scr. Mater., 48 (2003), 147.

10) M. F. Giordana, I. Alvarez-Armas, M. Sauzay and A. F. Armas: Key Eng. Mater., 465 (2011), 358.

11) D. T. Raske and J. M. Morrow: Mechanics of Materials in Low Cycle FatigueTtesting, in: Manual on Low Cycle Fatigue Testing ASTM STP 465, ASTM, Philadelphia, PA, (1969), 25.

12) A. Nagesha, M. Valsan, R. Kannan, K. Bhanu Sankara Rao and S. L. Mannan: Int. J. Fatigue, 24 (2002), 1285.

13) K. Guguloth, S. Sivaprasad, D. Chakrabarti and S. Tarafder: Mater. Sci. Eng. A, 604 (2014), 196.

14) D.-L. Wu, P. Zhao, Q.-Q. Wang and F.-Zh. Xuan: Inter. J. Fatigue, 70 (2015), 114.

15) ASM International: Fractography, ASM Handbook, Vol. 12, ASM International, OH, (1987), 857.

16) B. Fournier, M. Sauzay and A. Pineau: Inter. J. Plast., 27 (2011), 1803.

17) T. Sakai, A. Belyakov, R. Kaibyshev, H. Miura and J. J. Jonas: Prog. Mater. Sci., 60 (2014), 130.

18) V. Dudko, A. Belyakov, D. Molodov and R. Kaibyshev: Metall. Mat. Trans. A, 44 (2013), 162

19) A. Kipelova, R. Kaibyshev, A. Belyakov and D. Molodov: Mater. Sci. Eng. A, $\mathbf{5 2 8}$ (2011), 1280.

20) F. J. Humphreys and M. Hatherly: Recrystallization and Related Annealing Phenomena, Elsevier, UK, (2004), 624.

21) M. F. Giordana, P.-F. Giroux, I. Alvarez-Armas, M. Sauzay, A. Armas and T. Kruml: Mater. Sci. Eng. A, 550 (2012), 103.

22) G. E. Dieter: Mechanical Metallurgy, McGraw-Hill Book Company, London, UK, (1988), 631.

23) H. Semba and F. Abe: Energ. Mater., 1 (2006), 238. 